\title{
Above-light-line Nonlinear Surface Polaritons near a Conductive Interface: Threshold Case
}

\author{
Andrew D. Groza \\ Department of Computer Engineering, University “Ukraina”, 2Kotelnaya 22, Mykolaev, 54003, Ukraine \\ E-mail: amperslas@gmail.com
}

\begin{abstract}
We investigate the TM-polarized nonlinear surface polaritons (NLSP) propagating along a guided structure consisting of a magnetic optically linear medium and a nonmagnetic optically nonlinear medium with saturable permittivity separated by a flat conductive layer of zero thickness. We consider those values of hosting media bulk material parameters for which the NLSP existence (for zero sheet conductance) has threshold character with respect to the waves intensity. Based on the exact solution of Maxwell's equations we show that the energy and propagation properties of the NLSP near the above-light-line condition $(0<n<1)$ depend considerably on the surface conductivity of the layer, even the threshold character of the NLSP can be lost; for certain sheet conductance values these waves can exist in a linear limit. The NLSP propagation constant is defined by both the surface conductivity and field intensity and can be varied in a wide diapason, which gives an opportunity to obtain and control the important for quantum information processing $n \approx 0$ condition. For a chosen value of the NLSP propagation constant the NLSP field intensity and energy flux decrease when the surface conductivity grows; saturation of the nonlinear permittivity leads to an increase of the NLSP energy flux compared with Kerr-like nonlinearity. The results are potentially applicable for the development of tunable optical switches of the $n \approx 0$ condition in quantum photonic circuits.
\end{abstract}

\section{Introduction}

Surface plasmon polaritons (SPPs) are electromagnetic waves coupled to free electron oscillations traveling, typically, along a metal-dielectric interface [1]. The unique features the waves find promising applications in control and manipulation of light in nanometer scale [1,2]. Subwavelength localization of SPP field around the interface allows to reduce dimensions of plasmonic devices under diffraction limit [3]; strong enhancement of the SPP localized field enlarges nonlinear response of their guiding structure [4]; the SPP field distribution with peak at the interface leads to high sensitivity of the waves to any changes in the interface properties [5]. Surface polaritons were extensively investigated at the surfaces of dielectrics $[1,6]$, ferromagnetics [7], magnetic-semiconductor superlattices [8], metamaterials of hyperbolic [9] and nonlinear $[10,11]$ types, etc. A lot of SPP-based plasmonic devices and structures have been suggested and fabricated: modulators [12, 13], switches [13], waveguides [14], nanolasers [15], logic gates [16], chemical and biological sensors [17]. Control of the surface polaritons properties is a significant challenge in the creation of plasmonic devises; utilizing graphene in SPP guided structures seems to be the best answer [18]. Graphene is a two-dimensional (2D) material with a single layer of carbon atoms whose high conductivity at infrared frequencies combines with its unique property of being electrically or chemically tunable $[19,20]$. Recently proposed graphene plasmonic devices include modulators [21, 22], photodetectors [23], slow-light components [24], filters [25], polarizers [26], and biosensors [27]. Application of surface polaritons for mediating interemitter interactions in quantum information processing forms a novel and rapidly growing field of research [28, 29]. It includes realization of quantum interference between single plasmons in a nanoscale circuit [30], strong coupling between surface polaritons and quantum emitters [31] large Purcell enhancement [32], single plasmon generation [33] and detection [34]. Interactions efficiency of quantum emitters can be significantly increased in zero-index plasmonic structures. Stretching of the photon wavelength near the $n \approx 0$ condition enlarges the spatial scale of interemitter interactions and opens a way to control the interactions of quantum emitters which are separated at long distances with no need for their precise positioning [35-37].

Properties of surface polaritons are defined by the material parameters of their guiding structures. Recent progress in optical metamaterials confirm that every desirable value of their material parameters can be obtained, including negative and near-zero values of the magnetic permeability [38-47]. Magnetooptical metamaterials are created as substrates doped with metal nanoparticles of different shape and size [41-44] or low-loss all-dielectric structures [45]. Nonlinear metamaterials contain layers of metal and nonlinear media, or nonlinear media doped with metal nanoparticles [48]. The local field enhancement in the metamaterials can enlarge their nonlinearities and lead to saturation of the nonlinear permittivity. Surface polaritons in such artificially designed media obtain unexpected features.

Above-light-line surface polaritons whose phase speed exceeds the speed of light in vacuum can exist near the surface of an epsilon-near-zero (ENZ) metamaterial [49]. The effective refractive index of the polaritons is less than unity, the photon wavelength is larger and the phase advance 
is smaller than the same values for light in free space. Such properties lead to homogenization of the polaritons field distribution in the direction of propagation, which can be used to increase the interactions efficiency of quantum emitters in quantum information processing [37]. Contrary to the properties of bulk and guided electromagnetic waves in near-zero-index structures [35-37,50,51], for the surface polaritons in non-magnetic media, there exists a longwavelength cutoff [52] that is an obstacle in achieving the important for quantum information processing $n \approx 0$ condition. This difficulty can be overcome in magnetic media by a proper selection of the media dielectric permittivity and magnetic permeability values [53].

TM-polarized nonlinear surface polaritons (NLSP) can propagate along the surface of an optically nonlinear medium. Besides TM-polarized NLSP that exist in linear approximation there are ones whose existence becomes possible only in the nonlinear case and that have threshold character with respect to the NLSP field intensity or the NLSP energy flow. Threshold NLSP propagating along the interface of non-magnetic ENZ media obtain above-lightline features [54]. Implementation of conductive layers into waveguide structures can be used to provide tunable optical switches on deep-subwavelength scale. Plasmonic structure containing semi-infinite graphene sheet arrays embedded in the linear dielectric was proposed as a tunable optical switch whose threshold power is controlled by the chemical potential of graphene [55]; plasmonic structure with a single graphene monolayer demonstrates features of tunable optical bistable device with ultralow threshold value [56].

In this work we consider the threshold NLSP propagating along the guided structure consisting of a magnetic optically linear ENZ medium and a non-magnetic defocusing ENZ medium with saturable permittivity separated by a flat conductive layer of zero thickness. Based on the exact solution of Maxwell's equations we investigate the NLSP energy and propagation properties for the abovelight-line condition $0<n<1$, namely the dependences of the NLSP propagating constant vs. the NLSP total energy flux and the dependences of the NLSP propagating constant vs. the permittivity at the surface of the nonlinear medium for different values of sheet conductance for both saturable and Kerr-like nonlinearity.

\section{Statement of the problem}

We study the NLSP propagating in $x$-direction in the following lossless structure:

- $\quad$ semi-infinite optically linear magnetic medium (region $z<0$; dielectric permittivity $\varepsilon_{1}$, magnetic permeability $\left.\mu_{1}\right)$;

- $\quad$ conductive layer of zero thickness $(z=0$; sheet conductance $\sigma$ );

- $\quad$ semi-infinite optically nonlinear non-magnetic medium (region $z>0$; intensity-dependent permittivity $\varepsilon$ ).

The problem is assumed to be uniform in $y$-direction. The electric and magnetic NLSP fields are expressed by
$\vec{E}(\vec{r}, t)=\frac{1}{2}(\vec{E}(z) \exp i(k x-\omega t)+c . c),. \vec{E}(z)=\left(E_{x}, 0, E_{z}\right)$,

$$
\vec{H}(\vec{r}, t)=\frac{1}{2}(\vec{H}(z) \exp i(k x-\omega t)+c . c .), \vec{H}(z)=(0, H, 0) \text {. }
$$

The sheet conductance value of the layer is supposed to be purely imaginary: $\sigma=i \sigma^{\prime \prime}$ (it corresponds to graphene, at optical frequencies graphene is almost lossless and its conductivity can be considered a purely imaginary value [57]). The function which models the permittivity of the nonlinear medium is as follows:

$$
\varepsilon=\varepsilon_{x x}=\varepsilon_{z z}=\varepsilon_{0}+\varepsilon_{2}\left(|\vec{E}|^{2}\right)=\varepsilon_{0}+\frac{a\left(\left|E_{x}\right|^{2}+\left|E_{z}\right|^{2}\right)}{1+b\left(\left|E_{x}\right|^{2}+\left|E_{z}\right|^{2}\right)} \text {, }
$$

here $\varepsilon_{0}$ and $\varepsilon_{2}\left(|\vec{E}|^{2}\right)$ are the linear and the nonlinear parts of the permittivity. The quantity $b$ is supposed to be nonnegative; the signs of $\varepsilon_{0}, a, \varepsilon_{1}$, and $\mu_{1}$ are arbitrary. The saturation level of the intensity-dependent permittivity is $\varepsilon_{s}=\varepsilon_{0}+a / b$. The permittivity is Kerr-like for weak fields $\left(b|\vec{E}|^{2} \ll 1\right)$ and saturates for strong fields.

\section{Theory}

Maxwell's equations for the NLSP field components give:

$$
\begin{aligned}
& \frac{d H}{d \zeta}=i \varepsilon E_{x} \\
& n H=-\varepsilon E_{z} \\
& \frac{d E_{x}}{d \zeta}-i n E_{z}=i \mu H,
\end{aligned}
$$

where $n \equiv k / q$ is the dimensionless propagation constant and $\zeta \equiv z q$ is the dimensionless coordinate; $q=\omega / c$ is the wavevector of light in free space.

The solution of the system of Eq.(4a) to Eq.(4c) for the linear magnetic medium is as follows:

$$
\begin{aligned}
& H(\zeta)=H_{L}(0) \exp \left(k_{1} \zeta\right) \\
& k_{1}=+\left(n^{2}-\varepsilon_{1} \mu_{1}\right)^{1 / 2}
\end{aligned}
$$

here $H_{L}(0)$ is the magnitude of the NLSP magnetic field at the surface of the linear medium.

The solution of the system of Eq.(4a) to Eq.(4c) for the nonlinear medium (equations (2.10) and (2.13b) of 
[52]) gives the dependence $H=H(\zeta)$ by expressing the NLSP magnetic field $H$ in terms of dielectric permittivity $\varepsilon$, and the dimensionless coordinate $\zeta$ as an integral between the limits $\tilde{\varepsilon}$ and $\varepsilon$, where $\tilde{\varepsilon}$ is the dielectric permittivity at the surface of the nonlinear medium. The evident form of the relationship depends on $I\left(\varepsilon-\varepsilon_{0}\right)$ which is the inverse function of $\varepsilon_{2}\left(|\vec{E}|^{2}\right)$. Explicit forms of $I\left(\varepsilon-\varepsilon_{0}\right)$ and its integral $J\left(\varepsilon-\varepsilon_{0}\right)$ for the permittivity expressed by equation (3) are defined by Eqs. (9) and (10) from [58]:

$$
\begin{aligned}
& I\left(\varepsilon-\varepsilon_{0}\right)=\left(\varepsilon-\varepsilon_{0}\right)\left(a-b\left(\varepsilon-\varepsilon_{0}\right)\right)^{-1} \\
& J\left(\varepsilon-\varepsilon_{0}\right)=-\frac{1}{b}\left\{\varepsilon-\varepsilon_{0}+\left[\frac{a}{b}\right] \ln \left[1-\frac{b}{a}\left(\varepsilon-\varepsilon_{0}\right)\right]\right\} .
\end{aligned}
$$

For the case under consideration the tangential component of the NLSP electric field $E_{x}$ is continuous across the interface $z=0$, but the NLSP magnetic field has a gap whose value is proportional to the sheet conductance of the layer:

$$
H_{N L}(0)-H_{L}(0)=\sigma \frac{4 \pi}{c} E_{x}(0),
$$

where $H_{N L}(0)$ and $E_{x}(0)$ are the magnitudes of the NLSP magnetic and electric field components at the surface of the nonlinear medium.

Making use of the boundary condition one can obtain the equation for the NLSP propagation constant $n$ :

$$
\begin{aligned}
& k_{1}^{4}\left[\tilde{\varepsilon}\left(\tilde{\varepsilon} I\left(\tilde{\varepsilon}-\varepsilon_{0}\right)-J\left(\tilde{\varepsilon}-\varepsilon_{0}\right)\right) \frac{1}{\varepsilon_{1}}-I\left(\tilde{\varepsilon}-\varepsilon_{0}\right) \frac{2}{\varepsilon_{1}^{2}}\right]\left(\frac{4 \pi \sigma^{\prime \prime}}{c}\right)^{2}- \\
& k_{1}^{3}\left[\tilde{\varepsilon}\left(\tilde{\varepsilon} I\left(\tilde{\varepsilon}-\varepsilon_{0}\right)-J\left(\tilde{\varepsilon}-\varepsilon_{0}\right)\right) \frac{2}{\varepsilon_{1}}-I\left(\tilde{\varepsilon}-\varepsilon_{0}\right) \frac{4}{\varepsilon_{1}^{2}}\right]\left(\frac{4 \pi \sigma^{\prime \prime}}{c}\right)+ \\
& k_{1}^{2}\left[\tilde{\varepsilon}\left(\tilde{\varepsilon} I\left(\tilde{\varepsilon}-\varepsilon_{0}\right)-J\left(\tilde{\varepsilon}-\varepsilon_{0}\right)\right)\left\{1+\left(\frac{4 \pi \sigma^{\prime \prime}}{c}\right)^{2} \frac{\mu_{1}}{\varepsilon_{1}}+\frac{1}{\varepsilon_{1}^{2}}\right\}-\right. \\
& \left.I\left(\tilde{\varepsilon}-\varepsilon_{0}\right)\left\{2+\left(\frac{4 \pi \sigma^{\prime \prime}}{c}\right)^{2}\left(\frac{2 \mu_{1}}{\varepsilon_{1}}-\frac{\tilde{\varepsilon}}{\varepsilon_{1}^{2}}\right)\right\}\right]- \\
& k_{1}\left[\tilde{\varepsilon}\left(\tilde{\varepsilon} I\left(\tilde{\varepsilon}-\varepsilon_{0}\right)-J\left(\tilde{\varepsilon}-\varepsilon_{0}\right)\right) 2\left(\frac{4 \pi \sigma^{\prime \prime}}{c}\right) \mu_{1}-\right. \\
& I\left(\tilde{\varepsilon}-\varepsilon_{0}\right)\left\{4 \mu_{1}-2 \frac{\tilde{\varepsilon}}{\varepsilon_{1}}\right\}\left(\frac{4 \pi \sigma^{\prime \prime}}{c}\right) \mid+
\end{aligned}
$$

$$
\tilde{\varepsilon}\left(\tilde{\varepsilon} I\left(\tilde{\varepsilon}-\varepsilon_{0}\right)-J\left(\tilde{\varepsilon}-\varepsilon_{0}\right)\right) \varepsilon_{1} \mu_{1}-I\left(\tilde{\varepsilon}-\varepsilon_{0}\right)\left(2 \varepsilon_{1} \mu_{1}-\tilde{\varepsilon}\right)=0
$$

Roots of this four-order equation with respect to the positive $k_{1}=+\left(n^{2}-\varepsilon_{1} \mu_{1}\right)^{1 / 2}$ give the NLSP propagation constant as a function of both the dielectric permittivity at the surface of the nonlinear medium $\tilde{\varepsilon}$ and the sheet conductance value of the layer $\sigma^{\prime \prime}$.

For a zero-conductivity case eq. (9) transforms into the following expression:

$$
n^{2}=\frac{\varepsilon_{1} \tilde{\varepsilon}^{2}\left(\left(\mu_{1} \tilde{\varepsilon}-\varepsilon_{1}\right) I\left(\tilde{\varepsilon}-\varepsilon_{0}\right)-\mu_{1} J\left(\tilde{\varepsilon}-\varepsilon_{0}\right)\right)}{\tilde{\varepsilon}\left(\tilde{\varepsilon}^{2}-\varepsilon_{1}^{2}\right) I\left(\tilde{\varepsilon}-\varepsilon_{0}\right)-\left(\tilde{\varepsilon}^{2}+\varepsilon_{1}^{2}\right) J\left(\tilde{\varepsilon}-\varepsilon_{0}\right)} .
$$

The requirement for the NLSP field to vanish for $z \rightarrow+\infty$ and $z \rightarrow-\infty$ gives for polaritons in non-magnetic media a long-wavelength cutoff for the NLSP propagation constant $n^{2}>\max \left(0, \varepsilon_{0}, \varepsilon_{1}\right)$ which is valid in both linear and nonlinear cases [52]. In the case under consideration the cutoff obtains the following form:

$$
n^{2}>\max \left(0, \varepsilon_{0}, \varepsilon_{1} \mu_{1}\right)
$$

For both negative $\varepsilon_{0}$ and $\varepsilon_{1} \mu_{1}$ the cutoff obtains zero value, and the near-zero index condition $n \approx 0$ can be achieved.

The NLSP total energy flux per unit length in $y$ direction $Q$ consists of the energy flux in the linear medium $Q_{L}$ and the energy flux in the nonlinear medium $Q_{N L}$. The results of [52] allow expressing both these fluxes in terms of $\tilde{\varepsilon}$ :

$$
\begin{aligned}
& Q_{L}=\frac{c n}{16 \pi \varepsilon_{1}\left(n^{2}-\mu_{1} \varepsilon_{1}\right)^{1 / 2} q} \frac{\tilde{\varepsilon}}{\left(2 n^{2}-\tilde{\varepsilon}\right)}\left(\tilde{\varepsilon} I\left(\tilde{\varepsilon}-\varepsilon_{0}\right)-J\left(\tilde{\varepsilon}-\varepsilon_{0}\right)\right) \\
& ,(12) \\
& Q_{N L}=\operatorname{sgn}\left(\varepsilon_{\zeta}^{\prime}\right) \frac{c}{8 \pi} \frac{n}{q} \int_{\tilde{\varepsilon}}^{\varepsilon_{0}} d \varepsilon \frac{\varepsilon I\left(\varepsilon-\varepsilon_{0}\right)-J\left(\varepsilon-\varepsilon_{0}\right)}{\left(2 n^{2}-\varepsilon\right)[-2 V(\varepsilon)]^{1 / 2}}
\end{aligned}
$$

where

$$
V(\varepsilon)=-\frac{1}{2}\left[\frac{d H(\varepsilon)}{d \varepsilon}\right]^{-2}\left[\varepsilon^{2} I\left(\varepsilon-\varepsilon_{0}\right)-n^{2} H^{2}(\varepsilon)\right]
$$

and the expression for the NLSP magnetic field is as followes:

$$
H^{2}(\varepsilon)=\frac{\varepsilon}{2 n^{2}-\varepsilon}\left(\varepsilon I\left(\varepsilon-\varepsilon_{0}\right)-J\left(\varepsilon-\varepsilon_{0}\right)\right) .
$$

\section{Specific examples}

The calculations of the NLSP properties were carried out for the following values of parameters: 
- free-space wavelength: $\lambda=2 \pi c / \omega=1.06 \mu \mathrm{m}$;

- the linear medium: permittivity $\varepsilon_{1}=0.081$, permeability $\mu_{1}=-0.6$

- the nonlinear medium: linear part of permittivity $\varepsilon_{0}=$ 0.08 , Kerr coefficient $a=-4.4 \times 10^{-20} \mathrm{~m}^{2} / V^{2}$ (that is a typical value of nonlinear optical materials).

In linear approximation $(a=0)$ there are no surface polaritons for such material parameters (the squared linear propagation constant $n_{L}^{2}=\left(\varepsilon_{0} \varepsilon_{1}\left(\mu_{1} \varepsilon_{0}-\varepsilon_{1}\right)\right) /\left(\varepsilon_{0}^{2}-\varepsilon_{1}^{2}\right)$ [59] is negative), but their existence becomes possible in the nonlinear case and the corresponding NLSP has threshold behavior with respect to the NLSP energy flux and to the permittivity value at the surface of the nonlinear medium.

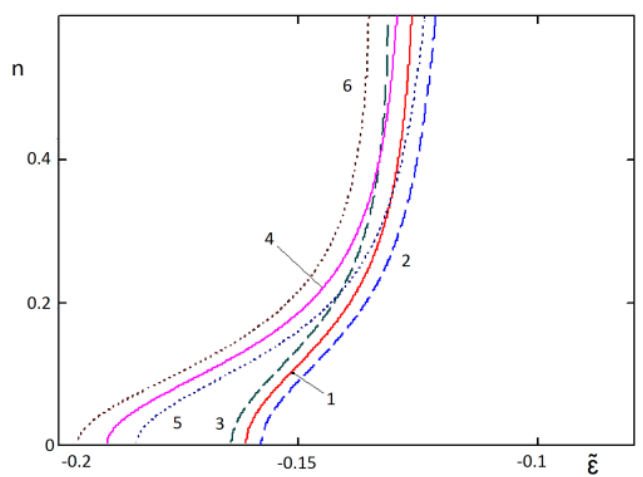

Figure 1. The NLSP propagation constant $\mathrm{n}$ vs. the permittivity at the surface of the nonlinear medium $\tilde{\varepsilon}$. The saturation level is $\varepsilon_{s}=-0.2$ : (1) $\sigma^{\prime \prime}=0$, (2) and (3) $\sigma^{\prime \prime}=-10^{-5} \mathrm{~S}$; the saturation level is $\varepsilon_{s}=-\infty$ : (4) $\sigma^{\prime \prime}=0$, (5) and (6) $\sigma^{\prime \prime}=-10^{-5} \mathrm{~S}$.

Figure 1 presents the dependences of the NLSP propagation constant vs. the permittivity at the surface of the nonlinear medium $\tilde{\varepsilon}$ for the saturation levels $\varepsilon_{s}=-0.2$ and $\varepsilon_{s}=-\infty$ for the sheet conductance values $\sigma^{\prime \prime}=0$ and $\sigma^{\prime \prime}=-10^{-5} \mathrm{~S}$ (a typical value for graphene at infrared frequencies [57]). For the case of zero conductivity the propagation constant values are defined by eq. (10) and there exists a single trace ((1) for $\varepsilon_{s}=-0.2$ and (4) for $\left.\varepsilon_{s}=-\infty\right)$ which originates from the $n=0$ and approaches infinity. The traces do not intersect the axis $n$, so such NLSP do not exist in linear limit. For the case $\sigma^{\prime \prime} \neq 0$ the propagation constant values are defined by eq.(9) and the abovementioned zero-conductivity traces are surrounded by the traces for $\sigma^{\prime \prime}=-10^{-5} \mathrm{~S}$ : ((2) and (3) for $\varepsilon_{s}=-0.2$; (5) and (6) for $\left.\varepsilon_{s}=-\infty\right)$. The traces (2) and (5) tend to a single point at the axis $n$, which gives the NLSP propagation constant value in the linear limit.

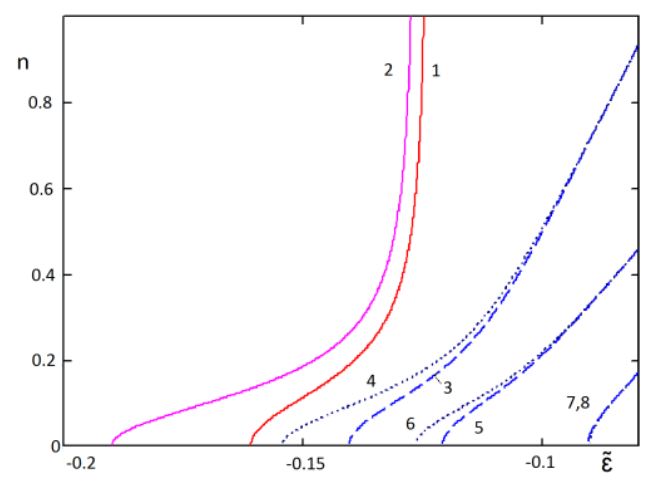

Figure 2. The NLSP propagation constant $n$ vs. the permittivity at the surface of the nonlinear medium $\tilde{\varepsilon}$. For the saturation level $\varepsilon_{s}=-0.2$ : (1) $\sigma^{\prime \prime}=0$, (3) $\sigma^{\prime \prime}=-6 \cdot 10^{-5}$ $\mathrm{S}$, (5) $\sigma^{\prime \prime}=-11 \cdot 10^{-5} \mathrm{~S}$, (7) $\sigma^{\prime \prime}=-18 \cdot 10^{-5} \mathrm{~S}$; for the saturation level $\varepsilon_{s}=-\infty$ : (2) $\sigma^{\prime \prime}=0$, (4) $\sigma^{\prime \prime}=-6 \cdot 10^{-5} \mathrm{~S},(6)$ $\sigma^{\prime \prime}=-11 \cdot 10^{-5} \mathrm{~S},(8) \sigma^{\prime \prime}=-18 \cdot 10^{-5} \mathrm{~S}$.

Figure 2 shows that with the increase of conductivity the corresponding intersection points approach smaller values of the propagation constant: traces (3) for $\varepsilon_{s}=-0.2$ and (4) for $\varepsilon_{s}=-\infty$ correspond to $\sigma^{\prime \prime}=-6 \cdot 10^{-5} \mathrm{~S}$; traces (5) for $\varepsilon_{s}=-0.2$ and (6) for $\varepsilon_{s}=-\infty$ correspond to $\sigma^{\prime \prime}=-11 \cdot 10^{-5} \mathrm{~S}$; traces (7) for $\varepsilon_{s}=-0.2$ and (8) for $\varepsilon_{s}=-\infty$ correspond to $\sigma^{\prime \prime}=-18 \cdot 10^{-5} \mathrm{~S}$. Figures 1 and 2 illustrate that NLSP properties depend considerably on the surface conductivity of the layer; for a certain value of sheet conductance the waves can exist even for near-zero field intensity.

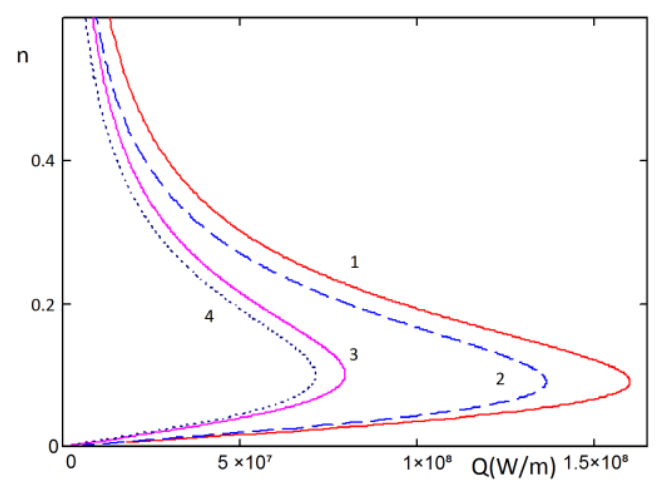

Figure 3. The NLSP propagation constant $n$ vs. the NLSP total energy flux Q. The saturation level is $\varepsilon_{s}=-0.2$ : (1) 


$$
\begin{aligned}
& \sigma^{\prime \prime}=0,(2) \sigma^{\prime \prime}=-10^{-5} \mathrm{~S} \text {; the saturation level is } \varepsilon_{s}=-\infty \\
& \sigma^{\prime \prime}=0, \text { (4) } \sigma^{\prime \prime}=-10^{-5} \mathrm{~S}
\end{aligned}
$$

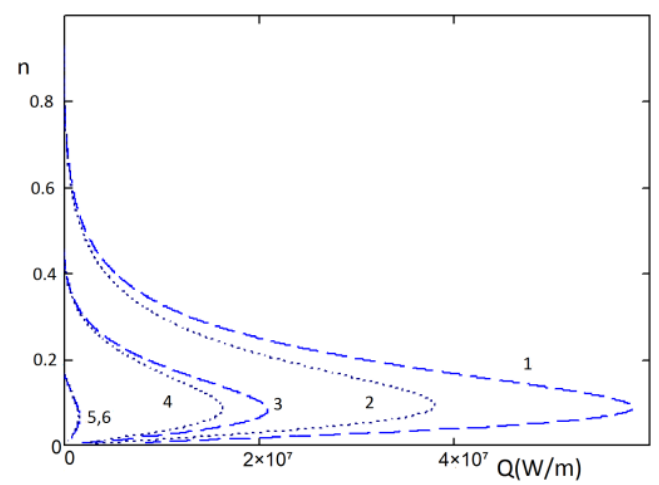

Figure 4. The NLSP propagation constant $n$ vs. the NLSP total energy flux $Q$. The saturation level is $\varepsilon_{s}=-0.2$ : (1) $\sigma^{\prime \prime}=-6 \cdot 10^{-5} \mathrm{~S}$, (3) $\sigma^{\prime \prime}=-11 \cdot 10^{-5} \mathrm{~S}$, (5) $\sigma^{\prime \prime}=-18 \cdot 10^{-5} \mathrm{~S}$; the saturation level is $\varepsilon_{s}=-\infty$ : (2) $\sigma^{\prime \prime}=-6 \cdot 10^{-5} \mathrm{~S}$, (4) $\sigma^{\prime \prime}=-11 \cdot 10^{-5} \mathrm{~S},(6) \sigma^{\prime \prime}=-18 \cdot 10^{-5} \mathrm{~S}$.

The dependences of the NLSP propagation constant vs. the NLSP energy flux $n=n(Q)$ are presented in Fig.3. The zero conductivity traces (1) for $\varepsilon_{s}=-0.2$ and (3) for $\varepsilon_{s}=-\infty$ correspond to the traces (1) and (4) from Figure 1. The traces for non-zero conductivity (2) for $\varepsilon_{s}=-0.2$ and (4) for $\varepsilon_{s}=-\infty$ correspond to traces (2) and (5) from Fig. 1. All the traces originate from $n=0$; the zero conductivity ones approach infinity, the non-zero conductivity ones intersect the axis $n$. Figure 4 presents the dependences $n=n(Q)$ for increased surface conductivity. The growth of the surface conductivity leads to a decease of the NLSP energy flux; the saturation of the nonlinear permittivity leads to growth of the NLSP energy flux compared with Kerr-like case.

The NLSP combine above-light-line features with threshold character of their excitation and are potentially applicable for the development of optical switch with tunable threshold level for application in quantum information processing circuits.

\section{Conclusion}

In this work we investigate the threshold NLSP propagating along the guided structure consisting of a magnetic optically linear ENZ medium and a non-magnetic optically nonlinear (defocusing) ENZ medium with saturable permittivity separated by a flat conductive layer of zero thickness. Based on the exact solution of Maxwell's equations we show that near the above-light-line condition $(0<n<1)$ the NLSP energy and propagation properties depend considerably on the sheet conductance value of the layer, even the threshold character of the NLSP can be lost and the NLSP can exist for vanishing field magnitude. The NLSP properties (and even their existence) can be controlled in two independent ways: by field intensity and by surface conductivity. The NLSP propagation constant can be varied in a wide diapason, from infinity to zero in the case of zero conductivity and from the certain finite value to zero in the case of non-zero conductivity, which gives an opportunity to obtain and control the important for quantum information processing $n \approx 0$ condition. For a chosen value of the NLSP propagation constant the NLSP field intensity and energy flux decease when the surface conductivity grows; saturation of the nonlinear permittivity leads to an increase of the NLSP energy flux compared with Kerr-like nonlinearity.

The results would provide potential application for tunable optical switching of the $n \approx 0$ condition in quantum information processing circuits.

\section{References}

[1] P. Berini, Long-range surface plasmon polaritons, Advances in Optics and Photonics, 1: 484-588, 2009.

[2] J. Zhang, L. Zhang, and W. Xu, Surface plasmon polaritons: physics and applications, Journal of Physics D: Applied Physics, 45: 113001-1-113001$19,2012$.

[3] D. Gramotnev, S. Bozhevolnyi, Plasmonics beyond the diffraction limit, Nature Photonics, 4: 83-91, 2010 .

[4] C. Argyropoulos, P.-Y. Chen, and A. Alù, Enhanced nonlinear effects in metamaterials and plasmonics, Advanced Electromagnetics, 1: 46-51, 2012.

[5] H. Lu, X. Gan, D. Mao, and J. Zhao, Graphenesupported manipulation of surface plasmon polaritons in metallic nanowaveguides, Photonics Research, 5(3): 162-167, 2017.

[6] A.V. Zayats, I.I. Smolyaninov, and A.A.Maradudin, Nano-optics of surface plasmon polaritons, Physical Reports, 408:131-314, 2005.

[7] D. Martin-Becerra, V.V. Temnov, T. Thomay, A. Leitenstorfer, R. Bratschitsch, G. Armelles, A. Garcia-Martin, and M.U. Gonzalez, Spectral dependence of the magnetic modulation of surface plasmon polaritons in noble/ferromagnetic/noble metal films, Physical Review B, 86: 035118-1035118-7, 2012. 
[8] V.R. Tuz, V.I. Fesenko, I.V. Fedorin, H.-B. Sun, and W. Hun, Coexistence of bulk and surface polaritons in a magnetic-semiconductor superlattice influenced by a transverse magnetic field, Journal of Applied Physics, 121:103102-1-103102-25, 2017.

[9] Q. Zhang, S. Zhou, S.F. Fu, and X. Z. Wang, Rich hybridized-polarization surface phonon polaritons in hyperbolic metamaterials, AIP Advances, 7:105211-1-105211-10, 2017.

[10] A.D. Groza, Above-light-line nonlinear surface polaritons near the surface of an epsilon-near-zero metamaterial, Journal of Modern Optics, 63(2):146-150, 2016.

[11]H.C. Zhang, Y. Fan, J. Guo, X. Fu, and T.J. Cui, Second-harmonic generation of spoof surface plasmon polaritons using nonlinear plasmonic metamaterials, ACS Photonics, 3(1):139-146, 2016.

[12] C. Jung, S. Yee, and K. Kuhn, Integrated optics waveguide modulator based on surface plasmon resonance, Journal of Lightwave Technology, 12: 1802-1806, 1994.

[13] T. Nikolajsen, K. Leosson, and S.I. Bozhevolnyi, Surface plasmon polariton based modulators and switches operating at telecom wavelengths, Applied Physics Letters, 85: 5833-5835, 2004.

[14] D. Zhang, K. Zhang, Q. Wu, X. Ding, and X. Sha, High-efficiency surface plasmonic polariton waveguides with enhanced low-frequency performance in microwave frequencies, Optics Express, 25(3): 2121-2129, 2017.

[15] W. Zhu, T. Xu, H. Wang, C. Zhang, P. B. Deotare, A. Agrawal, and H. J. Lezec, Surface plasmon polariton laser based on a metallic trench FabryPerot resonator, Science Advances, 3: 1700909-11700909-8, 2017.

[16]H. Wei, Z.X. Wang, X.R. Tian, M. Käll, and H. $\mathrm{Xu}$, Cascaded logic gates in nanophotonic plasmon networks, Nature Communications, 2:387-1-3875,2011

[17] K.M. Mayer, J.H. Hafner, Localized Surface Plasmon Resonance Sensors, Chemical Reviews, 111 (6): 3828-3857, 2011.

[18] S. Xiao, X. Zhu, B.-H. Li, N.A. Mortensen, Graphene-plasmon polaritons: From fundamental properties to potential applications, Frontiers of Physics, 11(2): 117801-1-117801-13, 2016.
[19] M. Liu, X. Yin, E. Ulin-Avila, B. Geng, T. Zentgraf, L. Ju, F. Wang, and X. Zhang, A graphene-based broadband optical modulator, Nature, 474(7349), 64-67, 2011.

[20] Y. Ding, X. Zhu, S. Xiao, H. Hu, L. H. Frandsen, N. A. Mortensen, and K. Yvind, Effective electrooptical modulation with high extinction ratio by a graphene-silicon microring resonator, Nano Letters, 15(7): 4393-4400, (2015).

[21]B. Sensale-Rodriguez, R. Yan, M. M. Kelly, T. Fang, K. Tahy, W. S. Hwang, D. Jena, L. Liu, and H. G. Xing, Broadband graphene terahertz modulators enabled by intraband transitions, Nature Communications, 3: 780-1-780-7, 2012.

[22] G. Liang, X. Hu, X. Yu, Y. Shen, L.H. Li, A.G. Davies, E. H. Linfield, H. K. Liang, Y. Zhang, S. F. $\mathrm{Yu}$, and Q. J. Wang, Integrated terahertz graphene modulator with $100 \%$ modulation depth, $A C S$ Photonics, 2(11): 1559-1556, 2015.

[23] L. Ju, B. Geng, J. Horng, C. Girit, M. Martin, Z. Hao, H. A. Bechtel, X. Liang, A. Zettl, Y. R. Shen, and F. Wang, Graphene plasmonics for tunable terahertz metamaterials, Nature Nanotechnoogy, 6(10): 630-634, 2011.

[24] R. Hao, X. Peng, E. Li, Y. Xu, J. Jin, X. Zhang, and H. Chen, Improved slow light capacity in graphenebased waveguide, Scientific Reports, 5: 15335-115335-9, 2015.

[25] B. Shi, W. Cai, X. Zhang, Y. Xiang, Y. Zhan, J. Geng, M. Ren, and J. Xu, Tunable band-stop filters for graphene plasmons based on periodically modulated graphene, Scientific Reports, 6: 26796 1-26796-6, 2016.

[26] Q. Bao, H. Zhang, B. Wang, Z. Ni, C. Lim, Y. Wang, D. Tang, and K. Loh, Broadband graphene polarizer, Nature Photonics, 5: 411-415, 2011.

[27] D. Rodrigo, O. Limaj, D. Janner, D. Etezadi, F. J. Garcia de Abajo, V. Pruneri, and H. Altug, Midinfrared plasmonic biosensing with graphene, Science, 349(6244): 165- 168, 2015.

[28] M.S. Tame, K. R. McEnery, S. K. Özdemir , J. Lee, S. A. Maier, and M. S. Kim, Quantum plasmonics, Nature Physics, 9: 329-340, 2013.

[29] L. Ming, C. Yang, and G. Guang-Can, Recent progress of the application of surface plasmon polariton in quantum information processing. Acta Physica Sinica, 66(14):144202-1-144202-10, 2017. 
[30] R.W. Heeres, L.P. Kouwenhoven, and V.Zwiller, Quantum interference in plasmonic circuits, Nature Nanotechnology, 8:719-722, 2013.

[31]P. Törmä, W. L. Barnes, Strong coupling between surface plasmon polaritons and emitters: a review, Reports on Progress in Physics, 78(1): 013901-1013901-34, 2015.

[32] A.Krasnok, S.Glybovski, and M.Petrov, Demonstration of the enhanced purcell factor in alldielectric structures, Applied Physics Letters, 108: 211105-1-211105-4, 2016.

[33] N. Somaschi, V. Giesz, L. De Santis, J. C. Loredo, M. P. Almeida, G. Hornecker, S. L. Portalupi, T. Grange, C. Anton, J. Demory, C. Gomez, I. Sagnes, N. D. Lanzillotti-Kimura, A. Lemaitre, A. Auffeves, A. G. White, L. Lanco, and P. Senellart, Near-optimal single-photon sources in the solid state, Nature Photonics, 10: 340-345, 2016.

[34] R. W. Heeres, S. N. Dorenbos, B. Koene, G.S. Solomon, L. P. Kouwenhoven, and V. Zwiller, On-Chip Single Plasmon Detection, Nano Letters, 10(2): 661-664, 2010.

[35] R. Sokhoyan, H.A. Atwater, Quantum optical properties of a dipole emitter coupled to an $\varepsilon$-nearzero nanoscale waveguide, Optics Express, 21(26): 32279-32290, 2013.

[36]E. Özg. n, E. Ozbay, H. Caglayan, Tunable ZeroIndex Photonic Crystal Waveguide for Two-Qubit Entanglement Detection, ACS Photonics, 3(11): 2129-2133, 2016.

[37] A.M. Mahmoud, I. Liberal, N. Engheta, Dipoledipole interactions mediated by epsilon-and-munear-zero waveguide supercoupling, Optical Materials Express, 7(2): 415-424, 2017.

[38] A.D. Boardman, V.V. Grimalsky, Y.S. Kivshar, S.V. Koshevaya, M. Lapine, N.M. Litchinitser, V.N. Malnev, M. Noginov, Y.G. Rapoport, and V.M. Shalaev, Active and tunable metamaterials, Laser \& Photonics Review, 5: 287-307, 2011.

[39] C.D. Giovampaola, N. Engheta, Digital metamaterials, Nature Materials, 13: 1115-1121, 2014.

[40] A.M. Urbas, Z. Jacob, L.D. Negro, N. Engheta, A.D. Boardman, P. Egan, A.B. Khanikaev, V. Menon, M. Ferrera, N. Kinsey, C. DeVault, J. Kim, V. Shalaev, A. Boltasseva, J. Valentine, C. Pfeiffer, A. Grbic, E. Narimanov, L. Zhu, S. Fan, A. Alù, E. Poutrina, N.M. Litchinitser, M.A. Noginov, K.F.
MacDonald, E. Plum, X. Liu, P.F. Nealey, C.R. Kagan, C.B. Murray, D.A. Pawlak, I.I.

Smolyaninov, V.N. Smolyaninova, and D. Chanda, Roadmap on optical metamaterials, Journal of Optics, 18: 093005-093058, 2016.

[41] A. Alù, A. Salandrino, N. Engheta, Negative effective permeability and left-handed materials at optical frequencies, Optics Express, 14(4): 1557-1567, 2006.

[42]H. Liu, H. Guo, L. Fu, S. Kaiser, H. Schweizer, and H. Giessen, Three-dimensional photonic metamaterial at optical frequences, Nature materials, 7: 31-37, 2008.

[43] B. Toal, M. McMillen, A. Murphy, W. Henderen, M. Arredondo, and R. Pollard, Optical and magnetooptical properties of gold core cobalt shell magnetoplasmonic nanowire arrays, Nanoscale, 6: 12905-129011, 2014.

[44] B. Toal, M. McMillen, A. Murphy, W. Henderen, P. Atkinson, and R. Pollard, Tuneable magnetooptical metamaterials based on photonic resonances in nickel nanorod arrays, Materials Research Express, 1: 015801-015812, 2014.

[45] K. Zhang, Q. Wu, J.-H. Fu, F.-Y. Meng, and L.-W. Li, Metamaterials With Tunable Negative Permeability Based on Mie Resonance, IEEE Transactions on Magnetics, 48 (11): 4289-4292, 2012.

[46] S. Kita, Y. Li, Ph. Munoz, O. Reshef, D.I. Vulis, R.W. Day, E. Mazur, and M. Lončar, On-chip alldielectric fabrication-tolerant zero-index metamaterials, Optics Express, 25(7): 8326-8334, 2017.

[47] R. Maas, J.Parsons, N.Engheta, and A.Polman, Experimental realization of an epsilon-near-zero metamaterial at visible wave lengths, Nature Photonics, 7: 907-912, 2013.

[48] K.P.M. Rishard, C. Li, T. Roger, M.Pietrzuk, A.D. Falco, D. Fassio, and M.R. Kaipurath, Z-scan characterization of epsilon-near-zero metamaterial, Proceedings of the conference PHOTON 14, Session 1; 2014 September 1-4; London.

[49] S. Feng, K. Halterman, Perfect Absorption in Ultrathin Epsilon-Near-Zero Metamaterials Induced by Fast-Wave Non-Radiative Modes, arXiv, 1112 0580.v1: 1-4, 2011.

[50] I. Liberal, N. Engheta, Near-zero refractive index photonics, Nature Photonics, 11:149-158, 2017. 
[51] E.J.R. Vesseur, T. Coenen, H. Caglayan, N. Engheta, and A. Polman, Experimental verification of $\mathrm{n}=0$ structures for visible light, Physical Review Letters, 110(1): 013902-1-013902-5, 2013.

[52] K.M. Leung, p-polarized nonlinear surface polaritons in materials with intensity-dependent dielectric functions, Physical Review B, 32: 5093 5101, 1985.

[53] A.D. Groza, Realizability of $n=0$ Condition for Nonlinear Surface Polaritons in Magnetic Media, Nonlinear Optics and Quantum Optics, 48: 265276, 2018.

[54] A.D. Groza, P-polarized Nonlinear Surface Polaritons Near the Surface of an Epsilon-NearZero Metamaterial with Saturable Permittivity: Threshold Cases, Nonlinear Optics and Quantum Optics, 47: 247-254, 2015.

[55]Z. Wang, B. Wang, H. Long, K. Wang, and P. Lu, Surface Plasmonic Lattice Solitons in Semi-infinite Graphene Sheet Arrays, Journal of Lightwave Technology, 35(14): 2960-2965, 2017.

[56] X. Dai, L. Jiang, and Y. Xiang, Low threshold optical bistability at terahertz frequencies with graphene surface plasmons, Scientific Reports, 5: 12271-1-12271-11, 2015.

[57]Z.K. Liu, Y.N. Xie, L. Geng, D.K. Pan, P. Song, Research of the method for measurement of graphen's carrier density, Romanian Journal of Physics, 61(5-6): 970-979, 2016.

[58] A.D. Groza, V.L. Strizhevskii, Properties of ppolarized nonlinear surface polaritons, Physica Status Solidi (b), 163: 381-388, 1991.

[59] Dmitruk N.L, LitovchenkoV.G, Strizhevskii V.L. Surface polaritons in semiconductors and dielectrics, 1st ed. Kiev: Naukova Dumka; p.13, 1989. 